\title{
Ueber Bildung von breiten Condylomen im äusseren Gehörgang.
}

\author{
Von \\ August Stöhr, \\ Assistenzarzt der medicin. Klinik in Würaburg.
}

Beobachtungen über Condylombildnng im äusseren Gehörgang sind, obgleich gowiss häufig genug gemacht, seither nur ganz vereinzelt zur Veröffentlichung gekommen, wie das aus der jüngsten $\mathrm{Zu}$. sammenstellung Schwartze's über Syphilis des Ohres *) ersichtlich ist. Abgesehen davon, dass der äussere Gehörgang Syphilitischer für gewrhnlich keiner eingebenden. Inspection unterzogen wird und augen. fallige subjective Beschwerden auch selten zir einer solchen auffordern, seheint man die Eruption von syphilitischen Plaques an genannter Stelle fiir eine zu unbedeutende Sache zu balten, um sie zum Gegenstande einer detaillirteren Besprechung zu machen. Seitdem ich nun vor fast drei Jahren zum ersten Male Gelegen. heit hatte, in rascher Aufeinanderfolge mehrere Spocinina dieser Erkrankung zu beobachten, bei denen die Ausbreitung und Grösse der Eruptionen zu den heftigsten Störungen fübrten, habe ich mich daran gewöbnt, in Fallen, in denen reichliche Eruption von Condylomen an den sonstigen Lieblingsstellen vorhanden war, genau das äussere Ohr zu besichtigen, und habe nun öfter Gelegenheit gehabt, die Gegenwart dieser Dermatitisform in Gehörgängen zu konstatiren. Eine

*) Dieses Archiv B. IV. H. 4 S. $253-271$. 
Reihe von vierzehn Beobachtungen, welche die verschiedensten Phasen der Erkrankung betreffen, setzt mich in den Stand, eine ziemlich vollstündige klinische Skizze derselben zu liefern. Blos drei meiner Kraxken gehörten dem männlichen Geschlechte an, und auch bei diesen waren nur leichte, gewissermassen rudimentäre Formen vorhanden. Mit Ausnahme von zweien zeigten dicse sämmtlichen Individuen eine aussergewöhnlich starke Verbreitung des Condylomausschlags an den Genitalien, den Oberschenkeln, unter den Brüsten. in der Achsel. höhle und den Zehenfurchen. Jeder Syphilidologe woiss, dass gewisse Individualitäten vorzugsweise zu dieser Reihe der Syphilisformen hinneigen, and dass diese Persönlichkeiten gewöhnlich solche sind. uej denen sehr fettreicher Panniculus vorhanden ist und die Secretion von Schweiss und Hauttalg in excessiver Weise stattindet. Schmutz und Vernachlässigung jeglicher Toilette der Haut neben den ehen erwähnten. Momenten haben auch in den ron mir beobacbteten Fällen gewiss den nächsten Anstoss zur Condylomeruption an der ungewöhulichen Stelle gegeben.

Den Justand, dass vorzugsweise der Gehörgang von Weibern befallen wurde, erkläre ich mir durch das fortwäbrendo Ueberdecktbleiben des Ohres bei gewissen Formen der. Haarfrisur, wodurch die Reinigung des Meatus externus erschwert und zur Aufstauung von Cerumen Veranlassung gegeben wird, Noch einfacher erklärt sicb dio Genese der nässenden Plaques im Gehörgange in zwei Fällen, in denen schon vor der syphilitischen Invasion längere. Zeit hindurch Otorrhoe vorhanden gewesen war und durch die anhaltende Maceration der Haut des Gebörganges das Zustandekommen der Syphilisform genügend vorbereitet war.

Auffallig ist mir geblieben, dass ich an der Ohrmuschel selbst nie eine Aufingerung von breiten Condylomen beobachtet habe; selbst das papulöse Syphilid, das in oft so reichlicher Eruption den behaarten Kopf befällt, gelangt bier nicht zur Entwicklung. Dieses Ausschliessungsverhaltniss scheint seine Begründung in dem histologischen Bau der Cutis an dieser Stelle zu haben, die bekanntlich arm an drüsigen Apparaten ist. (So hat man die Existenz von Schweissdritisen an dieser Hautpartie ganz geläugnet, bis Schueigger-Seidel ihr vereinzeltes Vorkommen nachwies.) Dagegen habe ich zweimal breite nässende Plaques unter dem Ohrläppchen und auf dasselbe übergreifend gesehen. unzweifelhaft. unter dem Einflusse eines Reizes. der durch Ohrsehmuck veranlasst war.

In weitaus der Mehrzahl der Fälle sah ich die condylomatösen Wucherungen in engen Gehörgängen eutstehen. Der Beginn der 
Affection fiel mit wenigen Ausnahmen mit der ersten Syphilizerkrankung des Individuums zusammen, gleichzeitig war meistens noch maculöses und papulöses Syphilid, starke Angina und beträchtliche allgemeine Drüsensch wellung rorhanden. Subjective Erscheinungen feblen im Anfang vollständig; die objective Untersuchung konstatirt häufig eine vermehrte Secretion von etwas verflüssigtem Cerumen, das in dichter leicht entfernbarer Schicht den Wandungen des Gehörgangs aufgelagert ist und oft das Lumen desselben in den engeren Partieen vollständig obstruirt. Die Spiegeluntersuejung ergibt nach geschehener Reinigung eine unbedeutende Schwellung der Gehörgangsauskleidung in den engeren Partieen, während das erste weitere Drittel sich noch vollständig intact verbäit. Die Färbung ist eine schmutzig-blaurothe, deutlich fleckige, häufig findet eine vermehrte Desquamation von Epidermisschüppchen statt, die auch in reichlicher Menge bei der mikroskopischen Untersuchung der Cerumenkrusten aufgefunden werden. In dieser Periode zeigen sich am Trommelfell noch keine Veränderungen, höchstens ein leichter Anflug von Injectionsröthe. Im weiteren Verlaufe erfährt nun die Schwellung und Röthung eine beträchtliche Zunahme, dic Secretion wird reichlicb, kleine Wattetampons, die eingeschoben werden, imbibiren sich in $z$ wei bis drei Stunden mit einer etwas übelriechenden Flüssigkeit, in der die Cerumenklumpohen bald vollständig fehlen und Eiterzellen in mässiger Menge nachgewiesen werden können; jene Flecke, an denen schon zu Anfang die Röthe an stärksten ausgesprochen war, zeigen sich immer deutlicher als flache Infiltrate, erheben sich ohne deutliche Abgrenzung uber das Niveau der umgebenden Hautpartieen und beeintrïchtigen dadurch das Lumen des Gebörganges. Einzelne meiner Kranken empfanden nun ein Gefühl von nicht besonders sehmerzhaftem Ziehen und Spannen und reagirten lebhafter gegen die Einfuhrung des Trichters, den man uun schon von schwächerem Kaliber wählen muss. Häufig erhält man eben nur noch ein Segment des Trommelfells zur Anschanung, die Grenze zwischen dem Rande des Trommelfells und der Gehörgangsendigung lässt sich nicht mehr genau bestimmen, das oberste Stratum des Trommelfells ist aufgelockert, mattroth, crscheint wie angehaucht, in drei Fällen unterschied ich deutlich schmutzig braunrothe, zienlich genau begrenzte Flecke; sonst bietet der Spiegelbefund für das Trommelfell nichts besonders Charakteristisches; das Verhalten ist rollständig dasselbe wie bei anderen Arten der Otitis externa. In Verlauf weniger lage und bei immer stärker wordender Secretion, die dann eine wirkliche Otorihoe darstellt, konmt endlich das volle Bild der Condylomerkrankung zur Entwicklung. Dio tieferen Partieen 
des Gehörgangs und das Trommelfell sind nun ganz der Babbethtung entzogen, indem die von den Wandungsn des Gahforgangs aus sich erhebenden Wucherungen anf einander trefferintend so vollständig seinen Querschnitt verlegen. Der Fingang zum Meatus und selbst Theile der Muschel zeigen nun durch die fortwährende Benetzung mit dem korrosiven Secret starke Röthung, stellenweise Vorlust der macerirten Epidermis. Nur in einem Fall sah ich die Condylome unmittelbar am Rand des Introitus aufsitzen; waren die Excrescenzen so weit vorgedrungen, so hatte mehr ein Herauswuchern derselben aus den eageren Partbieen stattgefunden. In Folge der Einengung der Neubildungen während ihres raschen Wachsthums durch den Widerstand der starren Gebörgangswandungen erlitten die Condylome Veränderungen in ibrer Gestalt, so dass sie bald die Form von kurzen Zapfen, bald von lappigen Bildungen darboten: keine auffallende Erscheinung ftur den Syphilidologen, der sonst oft Gelegenheit bat zu beobachten, wie das breite Condylom durch mechanische Bedingungen, die seiner topographischen Lage angehören, in seiner ursprünglichen Form verändert wird. Häufig kann man noch mit einer Sonde zwischen den aneinander liegenden Wucherungen eine karze Strecke eindringen, diese Manipulation ist aber meistens ron Blutungen und starkem Schmerrgefuhl gefolgt.

Die freien Flächen der condylonatoosen Auflagerungen zeigen ein verschiedenes Vorhalten. Meistens befinden sie sich im Zustande der Exulceration und es war mir wirklich die Thatsache auffallend, dass dieser Gewebszerfall fast aumahmslos ein sehr rascher war, wie er mit Ausnahme dor Genitalgegend anderwärts bei derartigen Eruptionen selten beobachtet wird. Diese Geschwüre sind leicht muldenförmig excavirt, an der unteren Circumferenz des Gehörgangs habe ich sie mehrmals von Rinnenform, rhagadenabnlich gesehen; die Riterung ist eine äusserst profuse, dex Geschwürsboden unzein, mit einer viseiden Eiterschicht bedeckt. In anderen Fallen; in denen das syphilitische Produkt mehr eine weniger umfangreiche aber derbe Papel darstellt, zeigt sich die Lpidermisschicht iber derselben verdickt, weisslich gefärbt, fein granulirt. Aber auch hier tritt bald stärkere Imbibition mit folgender Desquamation ein und es liegt nun das stark geröthete mit kleinen Höckern besetzte Corium leicht blutend bloss, oder die treie Fläche wird durch eine dünne gelbbraune Kruste überdeckt. Die Grösse der Condylome zeigt die verschiedensten Gradunterschiede; eng aneinander gelagert kleiden sie den Gebörgang vollständig aus und nur die facettirte Oberfläche solcher Lager lässt sie als durch Confinenz entstanden exkennen, die mehr vereinzelt stehenden sind von 
Erbsengrösse und darïber und erheben sich his zu 3 Millineter. Habeh sib eine solche beträchtlichere Grögse erreioht und stehen sie diaht gedringt, so wird dadureh der knorplige. Theil des Meatus wicht un* betrachtlich erweitert. Auf diesor Höhe der Erscheinungen kann sich nun die Affection, sofern keine Therapie einwirkr, Wochen hindurch erhalten; ich glazbe aber, dass atuch vollständig sich selbst überlassen, de das Condylom iaberkapt ja einen wenig stationären Charakter bat, almälig eine Involution oder im Anschlnss an die Geschwïrsbildung Yernarbung, wenn auch in sehr schleppender Weise, zu Stande kommt; es hestätigen mich in dieser Ansicht, dass diese Obrcondylome ninht selten obne alle Kunsthilfe heilen, die Ergebnisse der Untersuchung wou Syphilitischen, bei denen ich im Gehörgange narbige Stellen fand (Verlust des Follicularkörpers, Fehlen der Gohörgangthaare) und deren : Anamnese Aamn weiter ergab, „dass sie zu ciner gewissen Zeit etwass sohwerbörig gewesen seien, das Geftihl von Verstopftsein des äusieren Gehörganges gehabt hätten. Sie seien nun mit oinem Ohrlöffel eingegangen, hätten denselben aber nicht mehr sn weit wie früher rorfikren könnon. Darnach habe das $\mathrm{Ohr}$ längere Zeit goeitert." Dies ist die gewöbnaliche Beschreibung, die von dexartigen Kranken geliefert wird und ohne Hineinexaminiren wird man sich, wenn mán die Krankbeitsgeschichte Syphilitiscber nach dieser Richtung hin stwas genauer enforscht, die Ueberzeugung verschaffen; dass derartigge Vorgänge ziemlich bäufig sind.

In den von wir behandelten Fallen war häufig dic Iniseiturig einer Allgemeinbebandlung durch Inunction und lokale Reinigung binreichend, um rasch die. Involution der Condylome herbeizuführon; Waron ausgedehnte: Ulcerationen vorhanden, so war der Verlauf bin ziemlich tardiver, die Granulationsbildung durch Hämorrhagien häufig unterbrochen, Niobt uleerirte Plaques hinterliessen nach ihrem Schwinden nur einen lange Zeit sichtbaren Pigmentfleck; in jenen Fällen wo das Infiltrat zerfiel and die. Uloeration tiefere Hautschichten orgriff, blieb eine wenig pigmentirte Narbe zurïck, an der die Härchen fohlten und wabrscheinlich auch dio Ohrschmalzdrüsen zu Verlust ge: gangen waren; in einem Falle wenigstens, in dem ioh nach Monaten wieder eine Untersuchung vornahm, fand ich den Gehörgang liberhaupt trocken, die narbigen. Stellen aber von jeder Cerumenschicht vollständig frei.

Die subjectiven Störungen im Qefolge der beschriebenen Affection, aowohl was Schmerz, als was Functionsstörung anlangt, sind im Beginne unhedeutend und orreichen nur dann eine bedeutende Höhe, wenn die Fruption eine reiohliche, das Volum der Condylome ein 
beträchtliches ist. Der, Schinerz, im Beginne ein leiohtes Gefühl von Spannen, steigert sich, oobsild dio Exereseenzen den Canal ausfillen, mit fortdauerndem Wachsthum die Wandungen dehnen und auseinanderdritigen, und kann einen Grad erreichen, wie er bei Furunkelund Abscessbildung beobachtet wird. In zwei Fullen, in denon allerdings die Affection am stärksten entwickelt war, dauerte der heftigate prassende und bohrende Schmerz durch funf Tage hindurch an; eine dieser Kranken war vollständig schlaflos, jammerte laut und konnte nur durch starke Morphiuminjectionen beruhigt werden. Kaubewegungen vermehrten die Intensität des Schmerzes betrïchtlich, derselbe erstreckte sich auf Kopf und Nacken. Die fortwährende Jactation führte zu mässigen Fieberbewegungen (Puls 110, Temperatur 39,2), die wit dem Sohmarznachlass rasch schwanden. Trat die Affection einseitig auf oder war nur ein Gehörgang, was meistent der Fall war, vorzugsweise befallen, so schien das Hörvermögen nicht besonder' beeintrichtigt zu werden; eine genauere Untorsuchung ergab aber dennoch immer für die eine oder andere Seite eine sehr betrüchtliche Abnahme der Hörweite, die sich in zwei Fällen, in denen beide Gehörgänga fast vollständig verlegt waren, zu solcher Taubbeit steigerte, dass ich mich mit den Kranken nur schwer verständigen konnte. Einnal beobachtete joh Abschwächung des Gehörs in hohen. Grade zu einer Zeit, in der cben nur der orste Beginn der Affection sich nachwoisen liess, hier schien die Functionsstörung hauptsächlich dureh stärkeres Ergriffensein des Trommelfells bedingt zu sein, das stark geröthet und mit einer Eiterlage bedeckt war: Immerhin bleibt bei der Bourtheilung der Hörweite noch ein anderer Punkt zu berücksichtigen, der die Beurthejlung ersehwert, es ist dies der gleichzeitig varbandene Rachenkatarrh, der häufig bei Syphilitischen durch Schwellung am Ostium der Tube Schwerbörigkeit. verschiedenen Grades hervorruft. Mit der Involution der Condylome oder ihrer Vernarbung stellte sich die normale Hörweite wieder ein.

Wenn es sich auch als Regel zeigfe, dass mit den Schwinden der Plaques der gavzo Symptomencomplex sein Ende erreichte. so bestand doch in drei Fallen auch, nach der Tilgung der Syphilis noch lange Zeit eitriger Azusfluss, und die Auskleidung des Gehörgangs sowio das Trommelfell zeigten alle jene Veründerungen, wie sie durch eine lang andauernde Dermatitis hervorgerufen werden. Von sonstigen Folgexuständen der Condylombildung im äusseren Gehörgang erwähne ioh noch Verengorungen im knöchernen Theile. Der knorplige Theil ist die sthrkeren $\nabla$ ẹrengerungen ausgesetzt, öfter sogar wird er, mechanisch, wie oben benerkt, dureh das Hinein wucheru der Condylome 
erweitert, dagegen führt jene diffnse zellige Infiltration des Corium, wie sie in der Umgebung von breiten Condylomen überhaupt so bäufig geiroffen wird, im mittleren und letzten Drittel des Meatus leicht zu Stenosirungen, die anfänglich sogar einen höheren Grad haben, der von Einfluss auf die Functionirung sein muss, später allerdings eine beträchtliche Verringerung erfahren. Die ich gesehen habe waren alle gleichmässig ringförmig.

Recidiven der Condylombildung babe ich dreimal beobachtet. Im einen Falle bestand nach der ersten Attaque Otorrhoe fort und nach Verlauf ron 2 Nonaten kam es wiederholt zur Production von kleinen ziemlich consistenten Pupeln, die nicht ulcerirten und unter örtlicher Behandlung schwanden. In den beiden anderen schien dio Affection vullständig getilgt, bis nach Verlauf von 6 Wochen eine Syphilisrecidive sich durch Eruption eines squamösen Syphilids anzeigte und nun auch wieder Condylome im Gehörgange auftraten, die aber gleichfalls an $Z_{a h l}$ und Masse der ersten Generation nicht gleichkamen.

Die Diagnose der vorliegenden Erkrankungsform ist leicht, sobald nur einigermassen sorgfältig untersucht wird. Am ehesten wäre noch eine Verwechslung mit Polypen oder üppig wuchernden Granulationen möglich. Die Anamnese ist deshalb in zweifelhaften Fällen immer sorgfältig aufzunehmen, und der Nachweis der Syphilis durch Untersuchung der Genitalien, Fauces etc. zu liefern. Die Entscheidung zwischen Granulationen und condylomatösen Wucherungen dirfte bosonders in Fällen schwierig sein, in denen letztere zu lange andauernder Otorrhoe hinzutreten.

Was die Prognose anlangt, so ist dieselbe selbst für die ausge. sprochensten Fälle eine verhältnissmässig gïnstige in Bezug auf die Erhaltung des Gehörs; zurïckbleibende Otitis externa chronica und etwaige Verengerungen des Gebörganges verdienten jedoch entsprechende Berücksichtigung. Wird die Affection in ihrem Beginne erkannt, so ist kaum denkbar, dass bei entsprechender Therapie Formen aus ihr hervorgehen, wie ich sie nach wochenlanger Vernachlässigung beobachtet habe; wenn dagegen die histologischen Veränderungen einmal bis zu einem gewissen Grade gedieben sind, so ist der Verlauf immer ein sehr protrahirter, so dass Monate bis zur vollständigen restitutio in integrum vergehen.

Die Therapie erfordert vor allem eine antisyphilitisehe Beinandlung. Der Einfuss einer solchen (in meinen Fallen kam theils die Inunction, theils die Behandlung mit subcutanen Sublimatinjectionen zur Anwendung) ist so bedeutend, dass in leichteren Fillen ausser 
der nicht au ungehenden Reinlichkeitspflege durch Irrigation und Augspritzen jede weitere örtliche Behandlung umgangen wèdèn kann. Sind dagegen die Excrescenzen voluminös, der Schmerz und die Funotionsstörungen bedeutend, so ist dringend die Localtherapie indicirt. In wolcher W.eise dieselbe zur Ausfuhrung kommen sollte, hat mir in einigen Fällen viel Stoff zum Nachdenken gegeben. Selbstrerstänlich kann die etwas heroische Medication, wie sie bei Eruption von Condylomen an andoren Körperstellen gefahrlos in Anwendung gozogen wird, auf ein so irritables Organ, wic Gehörgang mit Trommelfell ist, nicht übertragen werden. Audcrerscits fordert wieder die Schwere des Symptomencomplexes und die Massenhaftigkeit der Neu. bildung auf, etwas mehr zu thun, als die Reihe der Adstringentien einzuspritzen, die gegen eine einfache Otitis recht gute Behelfe sein mögen, aber histologische Veränderungen ron solcher Schwere wie die Ohrcondylome gewiss nicht zum Schwinden bringen worden.

Vor Allem muss die Frage beantwortet werden : soll man ätzen, und womit? $\mathrm{Da}$ bei der Enge des Canals alle Manipulationen beträchtlich ersch wert sind, ja die wuchernde Neubildung denselben oft vollständig obstruirt, ist die A pplication des Aetzmittels wegen der erschwerten Begrenzung seiner gewebezerstörenden Wirkung mindestens bedenklich; denn eine starke Sublimatlösung, Chromsäure, Silbernitrat und andere Caustica, wie sie zur Zerstörung der Condylome meistens verwendet werden, in Contact mit dem Trommelfell könnten zur Perforation desselben, zu Mittelohrentziundung, überhaupt zu einer Reihe der schwersten Zufalle Veranlassung geben. Der auf die Aetzungen, sobald sie in der Tiefe des Gebörganges vorgenommen worden, folgende Schmerz erreicht ausserdem, wohl hauptsächlich in Folge der reactiven Entzündung in der Umgebung der Aetzstelle, eine so enorme Höhe, dass er allein schon als Contraindication betrachtet werden kann.

Wenn die Condylome als lappige oder zapfenförmige Wucherungen aus den engeren Partieen des Gehörganges hervordringen, so lassen sich manchmal wenigstens Bruchtheile derselben einfach durch dio Scheero entfernen; der noch festsitzende Rest geht dann häufig eine rasche Involution ein. Ist dies wie in den meisten Fallen nicht ausfubrbar, so muss man die allerdings ermüdende Aufgabe unteruchmen, in sehr häufigen Sitzungen peu a peu dureh vorsichtiges Touchiren mit dem Höllensteinstift Partikel um Partikel der Geschwulst zur Mortification zu bringen und in dieser Weise das Lumen des Gehörganges wiederherzustellen. Nebenbei muss in der scrupulösesten Weise das $\mathrm{Ohr}$ gereinigt werden; schwache Lösungen ron essigsaurem Zink und Kali hypermanganicum fand ich dazu am geeignetster. 